\title{
Atrial fibrillation in athletes
}

\author{
Derek Crinion MD, Adrian Baranchuk MD
}

Cite as: CMAJ 2020 January 13;192:E40. doi: 10.1503/cmaj.191209

CMAJ Podcasts: author interview at https://soundcloud.com/cmajpodcasts/191209-five

1 Too much exercise can cause atrial fibrillation

Large observational studies have shown that a sedentary lifestyle increases the risk of atrial fibrillation fivefold. ${ }^{1,2}$ However, high-intensity aerobic exercise, especially for men, also increases the risk of atrial fibrillation at a threshold of more than 1500-2000 lifetime hours. ${ }^{3}$ Evidence is less clear for women. ${ }^{3}$ In the majority of athletes, atrial fibrillation is paroxysmal and symptomatic., ${ }^{2,3}$

2 The causes of atrial fibrillation in athletes are multifactorial High-intensity aerobic exercise causes increased hemodynamic stress and generates free radicals. ${ }^{4}$ The atria appear to be particularly susceptible, with resultant dilatation, chronic inflammation and fibrosis. ${ }^{2-4}$ Autonomic modulation also plays a role, and athletes commonly describe triggers that are vagally (sleep, meals) or adrenergically (exercise) mediated. ${ }^{2,3}$

\section{3}

\section{Other causes of atrial fibrillation should be considered and}

\section{thromboembolic risk assessed}

It is important not to miss conditions associated with atrial fibrillation, including cardiomyopathies, channelopathies, pre-excitation, hyperthyroidism and alcohol or drug misuse., ${ }^{2,3}$ Energy supplements and performance enhancers should also be considered..$^{2,3}$ Data on stroke risk among athletes are limited, but the use of thromboembolic risk scores (CHADS$65)$ is recommended. $2,3,5$

Management of atrial fibrillation in athletes poses unique challenges

In nonrandomized studies, detraining has been shown to reduce atrial fibrillation, although athletes are often not willing to do this. ${ }^{2}$ Anti-arrhythmic medications are limited by bradycardia, impaired performance, long-term adverse effects and risk of ventricular arrhythmias during exercise., ${ }^{2,3} \mathrm{High}-$ impact sports are a contraindication to anticoagulation. ${ }^{2,3}$

\section{Ablation of atrial fibrillation is increasingly offered as first-line therapy}

Data remain limited to small, nonrandomized studies, but ablation is often the preferred option for symptomatic athletes. ${ }^{2,3}$ Despite the distinct pathophysiology between symptomatic athletes and nonathletes, success rates appear similar to those of nonathletes: about $60 \%$ for the first procedure and $>80 \%$ for the second. ${ }^{2,3}$ Athletes not meeting CHADS- 65 criteria must be informed of the need for anticoagulation for at least 2 months owing to the prothrombotic effect of catheter ablation. ${ }^{2,5}$

\section{References}

1. Qureshi WT, Alirhayim Z, Blaha MJ, et al. Cardiorespiratory fitness and risk of incident atrial fibrillation: results from the Henry Ford Exercise Testing (FIT) Project. Circulation 2015;131:1827-34.

2. Calkins H, Hindricks G, Cappato R, et al. 2017 HRS/EHRA/ ECAS/APHRS/SOLAECE expert consensus statement on catheter and surgical ablation of atrial fibrillation. Heart Rhythm 2017;14:e445-94.

3. Estes NAM III, Madias C. Atrial fibrillation in athletes: a lesson in the virtue of moderation. JACC Clin Electrophysiol 2017;3:921-8.

4. Guasch E, Benito B, Qi X, et al. Atrial fibrillation promotion by endurance exercise: demonstration and mechanistic exploration in an animal model. J Am Coll Cardiol 2013;62:68-77.

5. Andrade JG, Verma A, Mitchell LB, et al.; CCS Atrial Fibrillation Guidelines Committee. 2018 focused update of the Canadian Cardiovascular Society guidelines for the management of atrial fibrillation. Can J Cardiol 2018;34:1371-92.

\section{Competing interests: None declared}

This article has been peer reviewed.

Affiliation: Heart Rhythm Service, Kingston Health Sciences Centre, Queen's University, Kingston, Ont.

Correspondence to: Adrian Baranchuk, Adrian.Baranchuk@kingstonhsc.ca

CMAJ invites submissions to "Five things to know about ..." Submit manuscripts online at http://mc. manuscriptcentral.com/cmaj 